\title{
Combining therapy with recombinant human endostatin and cytotoxic agents for recurrent disseminated glioblastoma: a retrospective study
}

\author{
Jing-jing Ge ${ }^{1}$, Cheng Li ${ }^{1}$, Shao-pei Qi ${ }^{1}$, Feng-jun Xue ${ }^{1}$, Zhi-meng Gao ${ }^{1}$, Chun-jiang Yu ${ }^{2}$ and Jun-ping Zhang ${ }^{1 *}$ (D)
}

\begin{abstract}
Background: The optimal chemotherapeutics of recurrent disseminated glioblastoma has yet to be determined. We analyzed the efficacy and safety of recombinant human endostatin (rh-ES) combined with temozolomide and irinotecan in patients with recurrent disseminated glioblastoma.

Methods: We retrospectively reviewed 30 adult patients with recurrent disseminated glioblastoma treated with this combination chemotherapy at Department of Neuro-Oncology, Sanbo Brain Hospital, Capital Medical University of China from November 2009 to August 2018. Temozolomide was given orally at $200 \mathrm{mg} / \mathrm{m}^{2}$ daily for 5 days and rhES was administrated $15 \mathrm{mg} / \mathrm{d}$ daily for 14 days of each 28-day treatment cycle. Irinotecan was given intravenously every 2 weeks on a 28 -day cycle at $340 \mathrm{mg} / \mathrm{m}^{2}$ or $125 \mathrm{mg} / \mathrm{m}^{2}$ depending on antiepileptic drugs. Primary endpoint was progression-free survival (PFS) at 6 months ( $6 \mathrm{~m}$-PFS).

Results: The 6 m-PFS was $23.3 \%$. The median PFS was 3.2 months. The overall survival rate (OS) at 12 months was $28.6 \%$. The median OS was 6.9 months. Six out of $30(20 \%)$ patients demonstrated partial radiographic response and 11 (36.7\%) remained stable. The PFS of the 6 patients who got partial response was 5.8, 6.3, 6.9, 13.6, 15.8 and 16.6 months, respectively, and the median time interval of first response was 4 (range, 2.0-6.6) months. The most common adverse events were hematologic toxicities and gastrointestinal effects. The Grade $\geq 3$ adverse event was hematologic toxicities. The adverse events were manageable.
\end{abstract}

Conclusions: $\mathrm{Rh}-\mathrm{ES}$, in combination with cytotoxic drugs, was an alternative effective regimen with manageable toxicities in treatment of recurrent disseminated glioblastoma.

Keywords: Recurrent disseminated glioblastoma, Recombinant human endostatin, Temozolomide, Irinotecan, Chemotherapy

\section{Background}

Recurrent glioblastoma with extensive intracranial or spinal dissemination is refractory and have a poor prognosis. Re-irradiation is not always recommended due to the dose restriction, large tumor volume, tumor extensive locations, and late adverse events which included irreversible white matter changes and radionecrosis [1].

\footnotetext{
* Correspondence: doczhjp@hotmail.com

${ }^{1}$ Department of Neuro-Oncology, Sanbo Brain Hospital, Capital Medical University, No. 50, Yi-Ke-Song Road, Haidian District, Beijing 100093, People's Republic of China

Full list of author information is available at the end of the article
}

Chemotherapy is the primary treatment for those recurrent disseminated glioblastoma. However, there is no standard chemotherapy regimen currently. Anti-angiogenesis is a promising therapeutic strategy because that pathological angiogenesis is necessary for glioblastoma occurrence and metastasis. Bevacizumab, a humanized monoclonal antibody that inhibits vascular endothelial growth factor (VEGF), improved the progression-free survival (PFS), but had no effect on the overall survival (OS) [2-4]. Moreover, the effects of bevacizumab are transient and most patients' tumors progress just after a median time of 3-5 months [5-7]. Recombinant human endostatin (rh-ES) is an endogenous broad-spectrum

(c) The Author(s). 2020 Open Access This article is distributed under the terms of the Creative Commons Attribution 4.0 International License (http://creativecommons.org/licenses/by/4.0/), which permits unrestricted use, distribution, and reproduction in any medium, provided you give appropriate credit to the original author(s) and the source, provide a link to the Creative Commons license, and indicate if changes were made. The Creative Commons Public Domain Dedication waiver (http://creativecommons.org/publicdomain/zero/1.0/) applies to the data made available in this article, unless otherwise stated. 
angiogenesis inhibitor that has been shown to significantly improve therapeutic efficacy when combining with conventional chemotherapy agents in non-small-cell lung cancer, breast cancer and melanoma [8-10]. A previous study reported a case with recurrent brainstem pilocytic astrocytoma with neuraxis dissemination achieved a long-term tumor remission (more than 29 months) after receiving rh-ES in combination with conventional chemotherapy regimen of vincristine and carboplatin [11]. However, there has been no clinical experience reported on the use of rh-ES in glioblastoma treatment to date.

In this study, we retrospectively analyzed the effect and toxicity of rh-ES when combined with traditional cytotoxic drugs on adult recurrent disseminated glioblastoma.

\section{Methods}

\section{Patients selection}

We performed a retrospective observational study for all adult patients with recurrent disseminated glioblastoma treated with the combined regimens of temozolomide (TMZ), irinotecan (CPT-11) and rh-ES at Department of Neuro-Oncology, Sanbo Brain Hospital, Capital Medical University from November 2009 to August 2018.

Inclusion criteria were as follows: Age at 18-70 years old; Histological diagnosis of glioblastoma, the glioblastoma secondary to low-grade gliomas were also included; Recurrence was confirmed histologically or by radiographic evidence after surgery and adjuvant radiotherapy; Intracranial and/or spinal cord disseminated lesions at recurrence, whether lesions were disseminated at diagnosis was not limited; Treated with at least one cycle of the combined chemotherapy (TMZ, CPT-11 and rh-ES); Had at least one posttreatment radiographic follow-up. Patients who had previously received anti-angiogenic drug were also considered eligible. Patients with incomplete medical records were excluded.

\section{Treatment}

TMZ was given orally $200 \mathrm{mg} / \mathrm{m}^{2}$ for 5 days in each cycle. CPT-11 was administrated $125 \mathrm{mg} / \mathrm{m}^{2}$ for patients not receiving enzyme-inducing antiepileptic drugs (EIAEDs) and $340 \mathrm{mg} / \mathrm{m}^{2}$ for patients receiving EIAEDs on day 1 and day 15 . Rh-ES was administrated $15 \mathrm{mg} / \mathrm{d}$, daily for 14 days. One cycle of therapy was defined as 28 days. The clinical data collected included the following: Age, sex, previous therapies, number of relapse, treatment cycles, response, adverse events, subsequent treatments, progression date and dead date. The primary endpoint was PFS at 6 months (6 m-PFS). Secondary endpoints were median PFS, OS, OS at 12 months (12 $\mathrm{m}$-OS), objective response rate (ORR), disease control rate (DCR) and adverse events.

\section{Method of evaluation}

Contrast-enhanced MRI was performed at baseline and every 2 cycles thereafter until disease progression. Radiographic responses of the tumor were classified according to the RANO criteria [12]. A minimum of two largest lesions should be measured. For patients who have multiple lesions of which only one or two are increasing in size, the target enlarging lesion should be measured. Toxicities were classified according to Common Terminology Criteria for Adverse Events (CTCAE) 4.0.

\section{Statistical analysis}

Continuous variables were described by median and range, whereas categorical variables were described by numbers and percentages. The PFS and OS were analyzed using non-parametric Kaplan-Meier method. All statistical analyses were performed using SPSS 17.0. $p<$ 0.05 was considered to be statistically different.

\section{Results \\ Patient characteristics}

Between November 2009 and August 2018, 39 patients with recurrent glioblastoma were treated with the combined regimen. Excluding 9 patients whose tumors were not disseminated, 30 patients met the inclusion criteria with 21 men (70\%) and 9 women (30\%). The median age was 43 (range: 21-59). Patient characteristics are presented in Table 1. Five (16.7\%) patients' tumors were both intracranial and spinal disseminated and $14(46.7 \%)$ recurred more than once. Fourteen $(46.7 \%)$ patients received $>2$ previous regimens and 5 (16.7\%) received previous bevacizumab treatments. MGMT promoter was unmethylated in 9 patients and methylated in 4 patients. One patient harbored mutated IDH and 10 harbored wild-type IDH.

\section{Response to treatment}

Of the 30 patients, 6 achieved partial response, 11 got stable disease and 13 got progression disease. The ORR was $20 \%$ and DCR was $56.7 \%$. Figure 1 summarized the therapeutic effects of all the 30 patients. Seven of 30 patients received more than 4 cycles of chemotherapy, including 6 got partial response and 1 being in treatment. Three patients got progressed, but were still alive (red arrow). After progression, 8 patients received bevacizumab treatment (marked with asterisk).

Figure 2 showed the MRI changes of the patient 27. The tumor was located in right frontal lobe at diagnosis (Fig. 2a). After resection, radiotherapy and chemotherapy, the tumor was disappeared (Fig. 2b). However, 11 months after completion of the initial combined treatment, disseminated metastatic tumors occurred at frontal horn of the right lateral ventricles, the genu of corpus callosum and spinal (Fig. 2c, d and g). Then he received chemotherapy with TMZ, CPT-11 and rh-ES. After 4 months, the disseminated 
Table 1 Patient characteristics

\begin{tabular}{|c|c|}
\hline Patient characteristics & $r \mathrm{GBM}, n=$ \\
\hline \multicolumn{2}{|l|}{ Age, years } \\
\hline Mean & 41.8 \\
\hline Median & 43 \\
\hline Range & $21-59$ \\
\hline \multicolumn{2}{|l|}{ Sex, n (\%) } \\
\hline Male & $21(70.0)$ \\
\hline Female & $9(30.0)$ \\
\hline \multicolumn{2}{|l|}{ Initial KPS, n (\%) } \\
\hline $50-60$ & $8(26.7)$ \\
\hline $70-80$ & $16(53.3)$ \\
\hline $90-100$ & $6(20.0)$ \\
\hline \multicolumn{2}{|l|}{ Relapse, n (\%) } \\
\hline First & $16(53.3)$ \\
\hline Second & $7(23.3)$ \\
\hline Third & $7(23.3)$ \\
\hline \multicolumn{2}{|l|}{ Resection at last relapse before enrollment, n (\%) } \\
\hline Yes & $3(10.0)$ \\
\hline No & $27(90.0)$ \\
\hline \multicolumn{2}{|l|}{ Previous radiotherapy, $\mathrm{n}(\%)$} \\
\hline Yes & $30(100)$ \\
\hline No & $0(0)$ \\
\hline \multicolumn{2}{|l|}{ Previous radiation dose, $\mathrm{n}(\%)$} \\
\hline$>60 \mathrm{~Gy}$ & 5 \\
\hline 45-60Gy & 24 \\
\hline$<45 G y$ & 1 \\
\hline \multicolumn{2}{|l|}{ Number of previous chemotherapy regimens, n (\%) } \\
\hline 0 & $1(3.3)$ \\
\hline 1 & $2(6.7)$ \\
\hline 2 & $13(43.3)$ \\
\hline 3 & $7(23.3)$ \\
\hline$\geq 4$ & $7(23.3)$ \\
\hline \multicolumn{2}{|l|}{ Previous chemotherapy regimen, $\mathrm{n}(\%)$} \\
\hline Temozolomide, with concomitant radiotherapy & $25(83.3)$ \\
\hline Temozolomide & $21(70.0)$ \\
\hline Temozolomide + Cisplatin & $4(13.3)$ \\
\hline Temozolomide + Carboplatin & $1(3.3)$ \\
\hline Temozolomide + Apatinib & $4(13.3)$ \\
\hline Temozolomide + Bevacizumab & $2(6.7)$ \\
\hline Bevacizumab & $3(10.0)$ \\
\hline Temozolomide + Nimotuzumab & $1(3.3)$ \\
\hline Lomustine & $1(3.3)$ \\
\hline Nimustine & $1(3.3)$ \\
\hline Teniposide & $1(3.3)$ \\
\hline
\end{tabular}

Prior bevacizumab, n (\%)
Table 1 Patient characteristics (Continued)

\begin{tabular}{ll}
\hline Patient characteristics & $r \mathrm{GBM}, n=30$ \\
\hline Yes & $5(16.7)$ \\
No & $25(83.3)$ \\
Tumor dissemination, $\mathrm{n}(\%)$ & \\
Intracranial & $25(83.3)$ \\
Intracranial and spinal & $5(16.7)$ \\
MGMT status, n (\%) & \\
Unmethylated & $9(30)$ \\
Methylated & $4(13.3)$ \\
Not done/unknown & $17(56.7)$ \\
IDH1/2 mutation, $n$ (\%) & \\
Yes & $1(3.3)$ \\
No & $10(33.3)$ \\
Not done/unknown & $19(63.3)$ \\
EIAED & $0(0)$ \\
Non-EIAED & $30(100)$ \\
Patients alive & $4(13.3)$ \\
Patients not progressed & $1(3.3)$ \\
Median treatment length (cycles), range & $2(1-11)$ \\
\hline GBM glioblastoma, MGMT O6-methylguanine DNA-methyltransferase, IDH \\
isocitrate dehydrogenase, EIAED Enzyme-induced anti-epileptic drugs
\end{tabular}

tumors were significantly deceased and got a patial response (Fig. 2e, f and h). After 11 cycles, he discontinued the combined chemotherapy. However, 2 months later, he died from cerebral hernia.

\section{Survival}

At the last follow-up (March 31, 2019), 1 of 30 (3.3\%) patients were still not progressed and 4 (13.3\%) were still alive. The Kaplan-Meier curves of PFS and OS were showed in Fig. 3. The $6 \mathrm{~m}$-PFS was $23.3 \%$ (95\% CI, 8.2 to $38.4 \%)$. The median PFS was $3.2(95 \% \mathrm{CI}, 1.6$ to 4.8$)$ months (Fig. 3a). The $12 \mathrm{~m}$-OS was $28.6 \%$ (95\% CI, 12.1 to $45.1 \%)$. The median OS was $6.9(95 \% \mathrm{CI}, 3.8$ to 10.0$)$ months (Fig. 3b).

The PFS of the 6 patients who got partial response was $5.8,6.3,6.9,13.6,15.8,16.6$ months, respectively. The median time interval of first response was 4 (range, 2.0-6.6) months (Fig. 1). This demonstrated that the patients could achieve a long PFS once they got radiographic response in about 4 months.

Five of the 30 patients received previous bevacizumab treatment before enrollment. We analyzed the effect of previous bevacizumab on the survival time. The median PFS was 3.0 (95\%CI, 1.1 to 4.9 ) months versus 3.4 (95\%CI, 1.1 to 5.7$)$ months ( $\log \operatorname{rank} p=0.138)$ for the patients with previous bevacizumab treatment or not, respectively (Fig. 3c); the OS was 6.2 (95\%CI, 1.7 to 10.7) months versus $8.6(95 \% \mathrm{CI}, 5.7$ to 11.5$)$ months, respectively (Log rank 


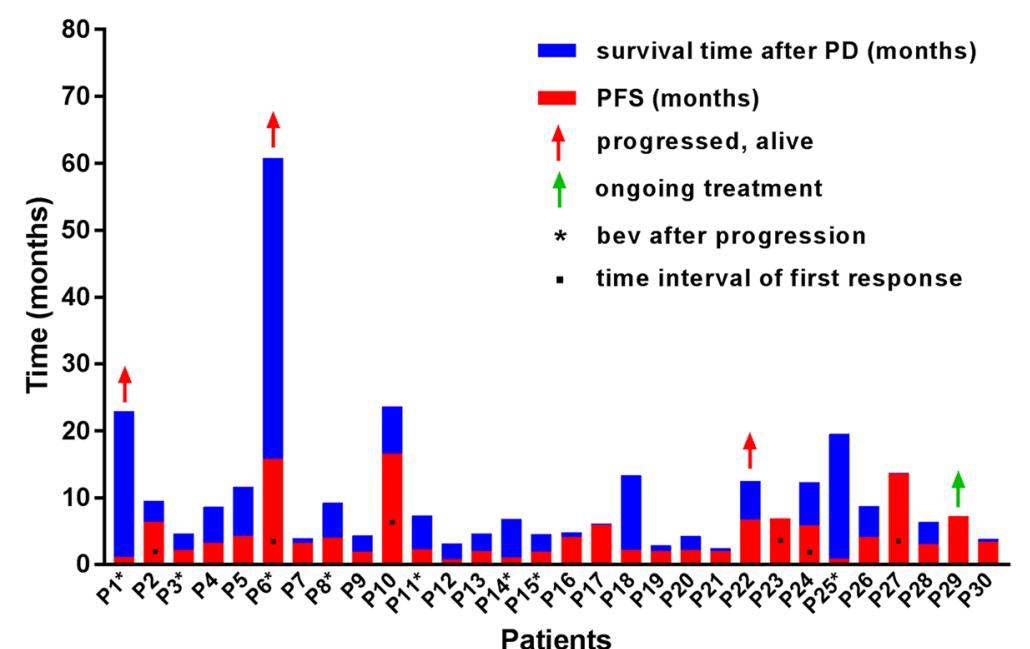

Fig. 1 Overview of the theraputic effects. Seven of 30 patients received more than 4 cycles of chemotherapy, including 6 patients got partial response (black spot) and 1 being in treatment (green arrow). The median time interval of first response was 4 (range, 2.0-6.6) months. Three patients got progressed, but not died (red arrow). After progression, 8 patients received bevacizumab treatment (marked with asterisk)

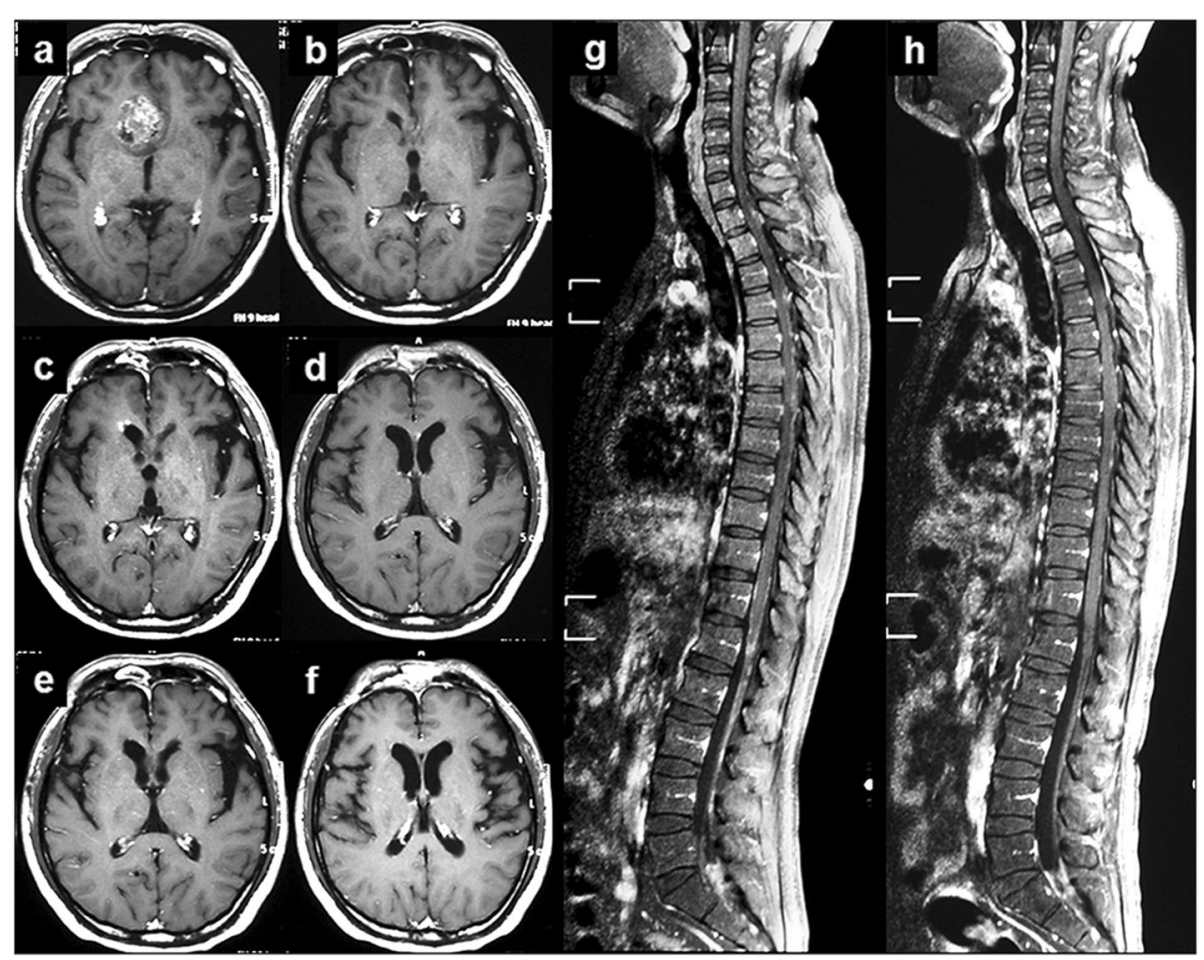

Fig. $2 \mathrm{MRI}$ of a typical case before and after treatment. a Evidence of a Gadolinium-enhanced lesion in the right frontal lobe before first surgery. b After surgery, chemoradiotherapy and adjuvant TMZ-based chemotherapy, the tumor got a complete response. c, $\mathbf{d}$ and $\mathbf{g}$ Eleven months after initial treatment completion, tumor recurrence was confirmed by MRl, which demonstrated widespread disseminated lesions in the frontal horn of right lateral ventricle, genu of corpus callosum and spine. $\mathbf{e}, \mathbf{f}$ and $\mathbf{h}$ After 4 months of combined chemotherapy, the tumors were significantly decreased 


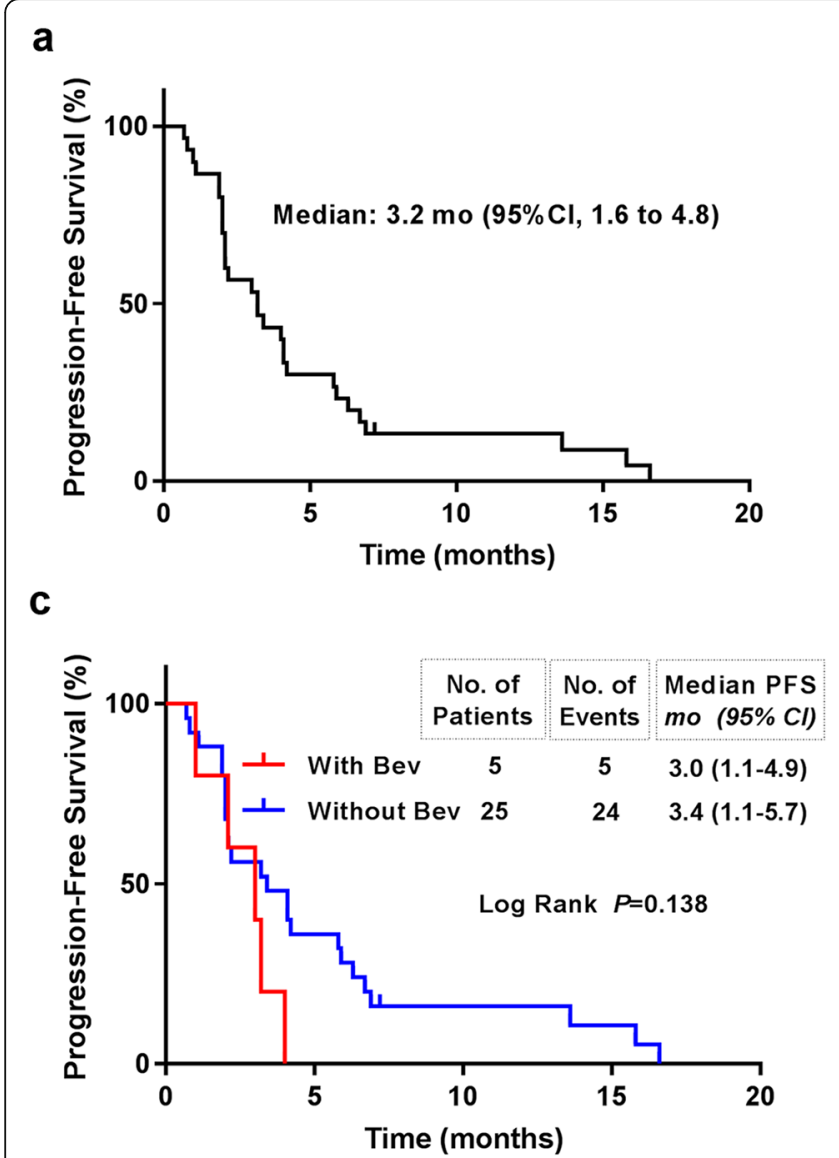

b

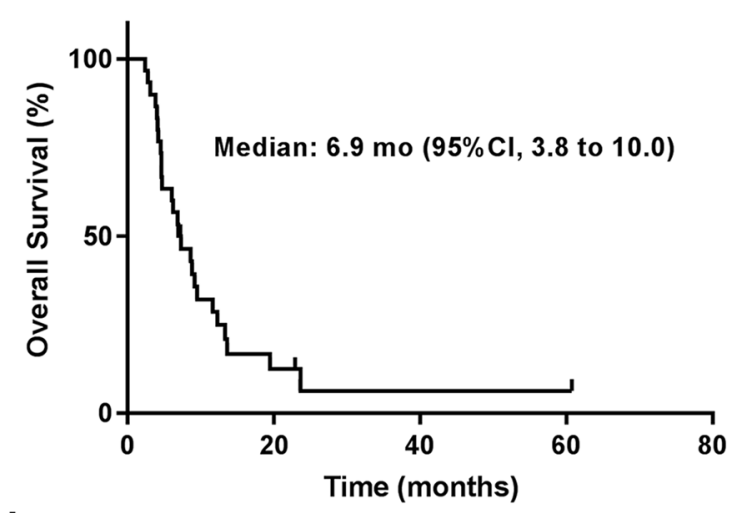

d

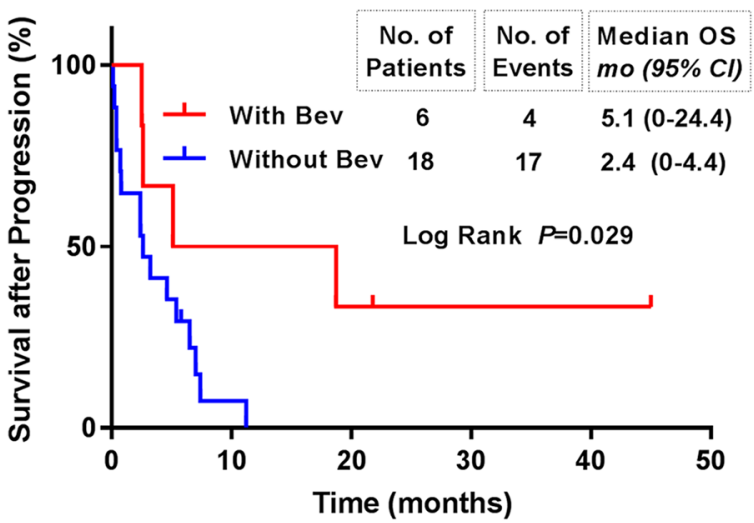

Fig. 3 Kaplan-Meier curves of Progression-Free Survival (PFS) and Overall Survival (OS). a PFS curve of all the enrolled patients; $\mathbf{b}$ OS curve of all the enrolled patients; $\mathbf{c}$ PFS curves of patients who received bevacizumab or not before enrollment. $\mathbf{d}$ OS curves of patients who received bevacizumab or not after tumor progression. Note: Bev, bevacizumab; mo, months

$p=0.098)$. This suggested that previous bevacizumab treatment may decrease the effect of combined chemotherapy though the difference was not statistical significant. This might be due to the small sample size.

After the tumor progression, 14 of the 29 patients received anticancer therapy, such as chemotherapy (12), radiotherapy (1) and surgery (1). Except for the 5 with previous bevacizumab, 6 out of 24 (25\%) patients received salvage bevacizumab treatment. The median survival time after progression of the patients with or without bevacizumab was 5.1 (95\%CI, 0 to 24.4$)$ months versus 2.4 (95\%CI, 0 to 4.9$)$ months, respectively (Log Rank $p=0.029)$ (Fig. 3d). This indicated bevacizumab treatment after progression from combined therapy may prolong the survival time.

We then investigated the relationship between chemotherapeutic effects and MGMT and IDH. The median PFS of the patients with unmethylated or methylated MGMT was 2.1 (95\%CI, 1.8 to 2.4 ) months versus 2 (95\%CI, 0 to 4.5 ) months, respectively (Log Rank, $p=0.92)$. The median PFS of the patients with mutated or wild-type IDH was 0.7 months versus 2 (1.8 to 2.2 ) months, respectively (Log Rank, $p=0.002)$. However, because more than half patients lost the information of IDH mutation status and MGMT methylation status, the results of the related PFS were not convincing which needed further study to confirm.

\section{Toxicity}

All the 30 patients were available for the safety analysis. There were no deaths from treatment-related toxicity. No one discontinued treatment due to adverse events. The most common adverse events (any grade) were hematologic toxicity $(22,73.3 \%)$ and gastrointestinal reactions $(11,36.7 \%)$, including nausea and vomiting $(8,26.7 \%)$, decreased appetite $(6,20 \%)$ and diarrhea (2, 6.7\%). The other adverse events included elevated aminotransferase $(5,16.7 \%)$, dizziness (2, 6.7\%), palpitation $(2,6.7 \%)$ and fatigue $(1,3.3 \%)$ (Table 2). Twelve out of $30(40 \%)$ patients experienced Grade $\geq 3$ adverse events: hematologic toxicity (12, 40\%) and elevated aminotransferase (1, 3.3\%). Dizziness and palpitation were mostly due to rh-ES and diarrhea was due to CPT-11. Dose reductions of CPT-11 or TMZ were occurred in 5 patients (16.7\%). Generally, the treatment was relatively well tolerated. 
Table 2 The toxicities of the combined chemotherapy of temozolomide, irinotecan and recombinant human endostatin

\begin{tabular}{lll}
\hline Toxicity & Any Grade (\%) & Grade 3 and $4(\%)$ \\
\hline Hematologic & $22(73.3)$ & $12(40.0)$ \\
Leukopenia & $22(73.3)$ & $11(36.7)$ \\
Neutropenia & $22(73.3)$ & $9(30.0)$ \\
Thrombocytopenia & $4(13.3)$ & $3(10.0)$ \\
Lymphocytopenia & $2(6.7)$ & $0(0)$ \\
Nonhematologic & & \\
Nausea and vomiting & $8(26.7)$ & $0(0)$ \\
Decreased appetite & $6(20.0)$ & $0(0)$ \\
Diarrhea & $2(6.7)$ & $0(0)$ \\
Elavated aminotransferase & $5(16.7)$ & $1(3.3)$ \\
Dizziness & $2(6.7)$ & $0(0)$ \\
Palpitation & $2(6.7)$ & $0(0)$ \\
Fatigue & $1(3.3)$ & $0(0)$ \\
\hline
\end{tabular}

\section{Discussions}

\section{Promising results of this rh-ES-combined chemotherapy}

The prognosis of recurrent disseminated glioblastoma is very poor with a mean OS of only 2 to 4 months even after kinds of treatments [13-15]. A recent retrospective study was conducted to examine the prognosis of glioma patients with leptomeningeal disease over 15-year period. It was demonstrated that 128 patients with glioblastoma had a median OS of $3.8(0.1-32.6)$ months after leptomeningeal disease diagnosis [16]. Improved therapeutic approaches are needed for recurrent glioblastoma with neuraxis dissemination.

In recent years, there are several reports on treatment of adult disseminated glioblastoma. Mandel JJ retrospectively reviewed 36 glioblastoma with leptomeningeal dissemination (including the newly-diagnosed) and assessed the impact of a variety of treatment modalities (hospice or radiation or chemotherapy). The survival time from the dissemination diagnosis was only 3.5 months. A combination of chemotherapy/targeted therapy and radiation had a significantly prolonged survival. However, for recurrent patients, re-radiation was always not recommended due to the dose restriction. In addition, this study did not focus on one chemotherapy regimen and fail to provide an effective therapeutic approach [17]. Most of the other published studies were case reports [18-20]. Okita Y reported a case of leptomeningeal dissemination of recurrent glioblastoma that achieved transient neurological and radiological improvement after chemotherapy with TMZ and bevacizumab (PFS $=2.3$ months) and died 5 months after diagnosis of dissemination [18]. In addition, a significant long-term remission ( $>2.5$ years) of disseminated glioblastoma to bevacizumab was observed in a patient with encephalocraniocutaneous lipomatosis.
However, the younger age (32-year-old) might influence the survival. Moreover, the biological differences between this particular glioblastoma in patient with encephalocraniocutaneous lipomatosis and common glioblastoma remain unknown [20].

Although the effect of CPT-11 combined with TMZ or bevacizumab in recurrent glioblastoma was investigated in sevaral studies, the status of dissemination of enrolled patients was not mentioned [21, 22].

The current study demonstrated a prolonged PFS and OS. In this study, after received combined chemotherapy regimen of rh-ES, TMZ and CPT-11, the $6 \mathrm{~m}-\mathrm{PFS}$ was $23.3 \%$, the median PFS and OS was 3.2 months and 6.9 months, respectively. The longest PFS was 16.6 months. This combined chemotherapy regimen was considered an effective salvage chemotherapy for recurrent disseminated glioblastoma.

\section{Possible mechanisms underlying the synergistic effects}

Different mechanisms, lack of overlapping major toxicities and the potent synergistic effect make these three drugs ideal candidates for combination chemotherapy. CPT-11, a topoisomerase I inhibitor, prevents re-ligation of DNA double strands and inhibits DNA replication, transcription and repair, resulting in tumor cell death. Toxicities of CPT-11 included diarrhea and relatively modest myelosuppression. CPT-11 could cross the blood brain barrier (BBB), which demonstrates excellent central nervous system penetration, but has shown only modest efficacy ( $6 \mathrm{~m}$-PFS was $15.7 \%)$ in patients with recurrent primary glioblastoma [23]. TMZ is a methylating agent that generates $\mathrm{O}^{6}$-methylguanine in DNA. TMZ is the standard therapy for patients with malignant glioma with myelosuppression as the main toxicity. Angiogenesis is a significant regulator of glioblastoma growth and tumor vascularity correlates with high-risk disease [24, 25]. Rh-ES is an endogenous broad-spectrum angiogenesis inhibitor, which could inhibit VEGF, cyclin D1, metalloproteinases, c-myc, integrins, and even Wnt signaling, thus inhibiting endothelial cell proliferation and migration, suppressing tumor vascularization and blocking the nutrition and oxygen supply to tumor cells [26-28]. The toxicity of rh-ES was mainly the modest cardiovascular toxicity.

The possible mechanisms underlying the synergistic effects were as follows: (1) Methylation of $\mathrm{O}^{6}$-guanine by TMZ may lead to recruitment of topoisomerase I and potentially enhance the probability of inducing CPT-11mediated damage. (2) The efficacy of gliomas treatments relies on drugs brain distribution through the $\mathrm{BBB}$, a monolayer of endothelial cells. In an in vivo model, TMZ and CPT-11 were shown that they could be transported by ATP-binding cassette B1 (ABCB1), the main efflux transporter expressed at the BBB level, which could influence the efficacy of TMZ and CPT-11 [29]. In a preclinical 
trial of NSCLC side population cells, rh-ES could prevent the migration of endothelial cells induced by VEGF and ABCB1 and inhibit the angiogenesis [30]. (3) Rh-ES decreases interstitial fluid pressure, promotes vascular normalization, thus induces a pressure gradient across the vasculature and improves drug penetration in tumors [31]. (4) The broad-sperum characteristic of rh-ES could help to reduce drug resistance and act synergistically with other cytotoxic drugs [8]. More direct evidences of the mechanism are needed to investigate in future preclinical studies.

\section{Postponing the use of bevacizumab and prolonging the survival time}

Bevacizumab may also aid in normalizing this disrupted tumor vasculature and improve drug delivery by facilitating uniform distribution of the vasculature [32-34]. Bevacizumab has been proved effective for recurrent glioblastoma.

Nonetheless most patients' tumors progress after a median time of 3-5 months or even during the bevacizumab administration [5-7]. The reasons of bevacizumab resistance might be the activation of other angiogenic pathways other than VEGF $[35,36]$. After progression on bevacizumab, there is no consensus on subsequent therapy, as multiple chemotherapy trials have failed to demonstrate discernible activity for salvage [37, 38]. Postponing the use of bevacizumab might be an alternative strategy.

In this current retrospective study, the patients could get radiographic response in a short time (4 months) and achieve a long PFS once getting response. Moreover, after tumor progression from the combined chemotherapy of rh-ES, TMZ and CPT-11, bevacizumab usage could help to prolong the survival time (5.1 months versus 2.4 months). The current combined regimen did not reduce the sensitivity of tumor to bevacizumab. This study provided an approach to postpone the use of bevacizumab and prolonging the survival time. The combined regimen of rh-ES, TMZ and CPT-11 was an alternative effective regimen prior to bevacizumab in treatment of recurrent disseminated glioblastoma.

\section{Conclusion}

In conclusion, the current study shows rh-ES combining TMZ and CPT-11 is an alternative effective regimen with manageable toxicities in treating recurrent disseminated glioblastoma. However, there are many further studies need to conduct: (1) large-scale prospective trials to investigate the effect of the combined chemotherapy regimens; (2) the mechanism underlying the synergistic effects.

\section{Abbreviations}

ABCB1: ATP-binding cassette B1; BBB: Blood brain barrier; CPT-11: Irinotecan; DCR: Disease control rate; EIAEDs: Enzyme-inducing antiepileptic drugs: ORR: Objective response rate; OS: Overall survival; PFS: Progression-free survival; rh-ES: Recombinant human endostatin; TMZ: Temozolomide; VEGF: Vascular endothelial growth factor

\section{Acknowledgments}

We thank our patients and their families. This work was presented as an abstract and a poster at the 14th meeting of the European Association of Neuro-Oncology (Neuro-Oncology, Volume 21, Issue Supplement_3, August 2019, Page NP, https://doi.org/10.1093/neuonc/noz126).

\section{Authors' contributions}

JPZ and CJY contributed to the conception and design of the work and revision of the manuscript. JJG contributed to data analysis and drafting the article. CL and ZMG contributed to data acquisition and statistical analysis. SPQ and FJX contributed to interpretation of the data and revision of the manuscript. All authors have read and approved the final manuscript and agreed to this information before submission.

\section{Funding}

This study was funded by Scientific Research Common Program of Beijing Municipal Commission of Education (Grant number: KM201710025027) and Neuro-oncology Research Program of Chinese Society of Neuro-oncology (Grant number: CSNO-2014-MSD02). The funding bodies had no role in the study design, data collection, data interpretation, or writing of this manuscript.

\section{Availability of data and materials}

The datasets used and/or analyzed during the current study are available from the corresponding author on reasonable request.

\section{Ethics approval and consent to participate}

This study was reviewed and approved by the ethics committee of Sanbo Brain Hospital Capital Medical University and the Department of NeuroOncology. Due to the retrospective nature without identifiable patient information, the requirement for informed consent was waived.

\section{Consent for publication}

Not applicable.

\section{Competing interests}

The authors declare that they have no competing interests.

\section{Author details}

${ }^{1}$ Department of Neuro-Oncology, Sanbo Brain Hospital, Capital Medical University, No. 50, Yi-Ke-Song Road, Haidian District, Beijing 100093, People's Republic of China. ${ }^{2}$ Department of Neurosurgery, Sanbo Brain Hospital, Capital Medical University, Beijing 100093, China.

Received: 24 June 2019 Accepted: 15 December 2019

Published online: 08 January 2020

\section{References}

1. Møller S, Munck Af Rosenschöld P, Costa J, et al. Toxicity and efficacy of reirradiation of high-grade glioma in a phase I dose- and volume escalation trial. Radiother Oncol. 2017;125(2):223-7.

2. Diaz RJ, Ali S, Qadir MG, et al. The role of bevacizumab in the treatment of glioblastoma. J Neuro-Oncol. 2017;133(3):455-67.

3. Wick W, Gorlia T, Bendszus M, et al. Lomustine and bevacizumab in progressive glioblastoma. N Engl J Med. 2017;377(20):1954-63.

4. Gilbert MR, Dignam JJ, Armstrong TS, et al. A randomized trial of bevacizumab for newly diagnosed glioblastoma. N Engl J Med. 2014;370(8):699-708.

5. Friedman HS, Prados MD, Wen PY, et al. Bevacizumab alone and in combination with irinotecan in recurrent glioblastoma. J Clin Oncol. 2009: 27(28):4733-40

6. Kreisl TN, Kim L, Moore K, et al. Phase II trial of single-agent bevacizumab followed by bevacizumab plus irinotecan at tumor progression in recurrent glioblastoma. J Clin Oncol. 2009;27(5):740-5.

7. Pope $\mathrm{WB}$, Xia Q, Paton VE, et al. Patterns of progression in patients with recurrent glioblastoma treated with bevacizumab. Neurology. 2011;76(5):432-7.

8. Zhao X, Su Y, You J, et al. Combining antiangiogenic therapy with neoadjuvant chemotherapy increases treatment efficacy in stage IIIA (N2) non-small cell lung cancer without increasing adverse effects. Oncotarget. 2016;7(38):62619-26. 
9. Chen J, Yao Q, Li D, et al. Neoadjuvant rh-endostatin, docetaxel and epirubicin for breast cancer: efficacy and safety in a prospective, randomized, phase II study. BMC Cancer. 2013;13:248.

10. Cui C, Mao L, Chi Z, et al. A phase II, randomized, double-blind, placebocontrolled multicenter trial of Endostar in patients with metastatic melanoma. Mol Ther. 2013;21(7):1456-63.

11. Ge JJ, Li C, Zhang JP. Long-term remission of recurrent brainstem pilocytic astrocytoma with neuraxis dissemination using recombinant human endostatin after failure of vincristine and carboplatin. World Neurosurg. 2018;1 10:397-402.

12. Wen PY, Macdonald DR, Reardon DA, et al. Updated response assessment criteria for high-grade gliomas: response assessment in Neuro-Oncology Working Group. J Clin Oncol. 2010;28(11):1963-72.

13. Arita N, Taneda M, Hayakawa T. Leptomeningeal dissemination of malignant gliomas. Incidence, diagnosis and outcome. Acta Neurochir (Wien). 1994;126:84-92.

14. Awad I, Bay JW, Rogers L. Leptomeningeal metastasis from supratentorial malignant gliomas. Neurosurgery. 1986;19:247-51.

15. Vertosick FT Jr, Selker RG. Brain stem and spinal metastases of supratentorial glioblastoma multiforme: a clinical series. Neurosurgery. 1990;27:516-22.

16. Andersen BM, Miranda C, Hatzoglou V, DeAngelis LM, Miller AM. Leptomeningeal metastases in glioma: The Memorial Sloan Kettering Cancer Center experience. Neurology. 2019;92(21):e2483-91.

17. Mandel JJ, Yust-Katz S, Cachia D, et al. Leptomeningeal dissemination in glioblastoma; an inspection of risk factors, treatment, and outcomes at a single institution. J Neuro-Oncol. 2014;120(3):597-605.

18. Okita Y, Nonaka M, Umehara T, et al. Efficacy of temozolomide and bevacizumab for the treatment of leptomeningeal dissemination of recurrent glioblastoma: a case report. Oncol Lett. 2015;9(4):1885-8.

19. Kong X, Wang Y, Liu S, et al. Brain stem and entire spinal leptomeningeal dissemination of supratentorial glioblastoma multiforme in a patient during postoperative radiochemotherapy: case report and review of the literatures. Medicine (Baltimore). 2015;94(24):e962.

20. Fukaya R, Ozaki M, Kamamoto D, et al. Significant antitumor response of disseminated glioblastoma to bevacizumab resulting in long-term clinical remission in a patient with encephalocraniocutaneous lipomatosis: a case report. Mol Clin Oncol. 2016;5(4):417-21.

21. Reynés $G$, Martínez-Sales $V$, Vila $V$, Balañá $C$, Pérez-Segura $P$, Vaz MA, et al. Phase II trial of irinotecan and metronomic temozolomide in patients with recurrent glioblastoma. Anti-Cancer Drugs. 2016;27(2):133-7.

22. Gilbert MR, Pugh SL, Aldape K, et al. NRG oncology RTOG 0625: a randomized phase II trial of bevacizumab with either irinotecan or dose-dense temozolomide in recurrent glioblastoma. J Neuro-Oncol. 2017;131(1):193-9.

23. Prados MD, Lamborn $K$, Yung WKA, et al. A phase 2 trial of irinotecan (CPT11) in patients with recurrent malignant glioma: a North American Brain Tumor Consortium study. Neuro-Oncology. 2006;8:189-93.

24. Ribatti D, Surico G, Vacca A, et al. Angiogenesis extent and expression of matrix metalloproteinase-2 and -9 correlate with progression in human neuroblastoma. Life Sci. 2001;68(10):1161-8.

25. Peddinti $R$, Zeine $R$, Luca $D$, et al. Prominent microvascular proliferation in clinically aggressive neuroblastoma. Clin Can Res. 2007;13(12):3499-506.

26. Abdollahi A, Hlatky L, Huber PE, et al. Endostatin: the logic of antiangiogenic therapy. Drug Resist Updat. 2005;8(1-2):59-74

27. Hanai J, Gloy J, Karumanchi SA, et al. Endostatin is a potential inhibitor of Wnt signaling. J Cell Biol. 2002;158(3):529-39.

28. Ling $Y$, Yang $Y$, Lu N, et al. Endostar, a novel recombinant human endostatin, exerts antiangiogenic effect via blocking VEGF-induced tyrosine phosphorylation of KDR/Flk-1 of endothelial cells. Biochem Biophys Res Commun. 2007;361(1):79-84.

29. Goldwirt L, Beccaria K, Carpentier A, et al. Irinotecan and temozolomide brain distribution: a focus on ABCB1. Cancer Chemother Pharmacol. 2014;74(1):185-93.

30. Cao B, Jia J, Ma L, et al. Recombinant human endostatin could eliminate the pro-angiogenesis priority of SP cells sorted from non-small cell lung cancer cells. Clin Transl Oncol. 2012;14(8):575-85.

31. Tong RT, Boucher Y, Kozin SV, et al. Vascular normalization by vascular endothelial growth factor receptor 2 blockade induces a pressure gradient across the vasculature and improves drug penetration in tumors. Cancer Res. 2004;64(11):3731-6.

32. Folkman J. Angiogenesis: an organizing principle for drug discovery? Nat Rev Drug Discov. 2007;6:273-86.

33. Jain RK. Normalization of tumor vasculature: an emerging concept in antiangiogenic therapy. Science. 2005;307:58-62.

34. Jain RK, Duda DG, Clark JW, et al. Lessons from phase III clinical trials on anti-VEGF therapy for cancer. Nat Clin Pract Oncol. 2006;3:24-40.
35. Goldwirt L, Beccaria K, Carpentier A, et al. Preclinical impact of bevacizumab on brain and tumor distribution of irinotecan and temozolomide. J NeuroOncol. 2015;122:273-81.

36. Batchelor T, Sorensen AG, di Tomaso E, et al. AZD2171, a pan-VEGF rreceptor tyrosine kinase inhhibitor, normalizes tumor vasculature and alleviates edema in glioblastoma patients. Cancer Cell. 2007;11(7):83-95.

37. Rahman R, Hempfling K, Norden AD, et al. Retrospective study of carmustine or lomustine with bevacizumab in recurrent glioblastoma patients who have failed prior bevacizumab. Neuro-Oncology. 2014;16(11):1523-9.

38. Chamberlain MC, Kim BT. Nivolumab for patients with recurrent glioblastoma progressing on bevacizumab: a retrospective case series. J Neuro-Oncol. 2017;133(3):561-9.

\section{Publisher's Note}

Springer Nature remains neutral with regard to jurisdictional claims in published maps and institutional affiliations.
Ready to submit your research? Choose BMC and benefit from:

- fast, convenient online submission

- thorough peer review by experienced researchers in your field

- rapid publication on acceptance

- support for research data, including large and complex data types

- gold Open Access which fosters wider collaboration and increased citations

- maximum visibility for your research: over $100 \mathrm{M}$ website views per year

At BMC, research is always in progress.

Learn more biomedcentral.com/submissions 